\title{
Distinct Regions within Medial Prefrontal Cortex Process Pain and Cognition
}

\author{
๑DAndrew Jahn, ${ }^{1}$ ๑Derek Evan Nee, ${ }^{2}$ William H. Alexander, ${ }^{3}$ and Joshua W. Brown ${ }^{4}$ \\ ${ }^{1}$ Haskins Laboratories, New Haven, Connecticut 06511, ${ }^{2}$ Department of Psychology, Florida State University, Tallahassee, Florida 32312-4301, ${ }^{3}$ Department \\ of Experimental Psychology, Ghent University, B-9000 Ghent, Belgium, and ${ }^{4}$ Department of Psychological and Brain Sciences, Indiana University, \\ Bloomington, Indiana 47405
}

\begin{abstract}
Neuroimaging studies of the medial prefrontal cortex (mPFC) suggest that the dorsal anterior cingulate cortex (dACC) region is responsive to a wide variety of stimuli and psychological states, such as pain, cognitive control, and prediction error (PE). In contrast, a recent meta-analysis argues that the dACC is selective for pain, whereas the supplementary motor area (SMA) and pre-SMA are specifically associated with higher-level cognitive processes (Lieberman and Eisenberger, 2015). To empirically test this claim, we manipulated effects of pain, conflict, and PE in a single experiment using human subjects. We observed a robust dorsal-ventral dissociation within the $\mathrm{mPFC}$ with cognitive effects of PE and conflict overlapping dorsally and pain localized more ventrally. Classification of subjects based on the presence or absence of a paracingulate sulcus showed that PE effects extended across the dorsal area of the dACC and into the pre-SMA. These results begin to resolve recent controversies by showing the following: (1) the mPFC includes dissociable regions for pain and cognitive processing; and (2) meta-analyses are correct in localizing cognitive effects to the dACC, although these effects extend to the pre-SMA as well. These results both provide evidence distinguishing between different theories of MPFC function and highlight the importance of taking individual anatomical variability into account when conducting empirical studies of the mPFC.
\end{abstract}

Key words: cognition; conflict; dorsal anterior cingulate cortex; medial prefrontal cortex; pain; paracingulate sulcus

\section{Significance Statement}

Decades of neuroimaging research have shown the mPFC to represent a wide variety of stimulus processing and cognitive states. However, recently it has been argued whether distinct regions of the mPFC separately process pain and cognitive phenomena. To address this controversy, this study directly compared pain and cognitive processes within subjects. We found a double dissociation within the MPFC with pain localized ventral to the cingulate sulcus and cognitive effects localized more dorsally within the dACC and spreading into the pre-supplementary motor area. This provides empirical evidence to help resolve the current debate about the functional architecture of the mPFC.

\section{Introduction}

The medial prefrontal cortex (mPFC) ranks among the most widely studied regions of the brain because of its involvement in various aspects of cognitive control and reinforcement learning.

Received July 7, 2016; revised Sept. 19, 2016; accepted Sept. 20, 2016.

Author contributions: A.J., D.E.N., W.H.A., and J.W.B. designed research; A.J. performed research; A.J. analyzed data; A.J. and D.E.N. wrote the paper.

This work was supported in part by the Indiana METACyt Initiative of Indiana University, funded in part through Lilly Endowment, Inc (major grant), and National Institutes of Health, National Institute of Child Health and Human Development Grant HD 073288 to Principal Investigator Julie van Dyke. The content is solely the responsibility of the authors and does not represent the official views of the National Institutes of Health. We thank Sean Berry and Colleen McCracken for help with scanning.

The authors declare no competing financial interests.

Correspondence should be addressed to Dr. Joshua W. Brown, Department of Psychological and Brain Sciences, 1101 E Tenth Street, Bloomington, IN 47405. E-mail: jwmbrown@indiana.edu.

DOI:10.1523/JNEUROSCI.2180-16.2016

Copyright $\odot 2016$ the authors $\quad 0270-6474 / 16 / 3612385-08 \$ 15.00 / 0$
In particular, the mPFC is responsive to conflict (Botvinick et al., 2001), error detection (Gehring et al., 1993), error likelihood (Brown and Braver, 2005), pain (Rainville et al., 1997), time on task (Grinband et al., 2011), and volatility (Behrens et al., 2007). We and others have proposed that these effects and others can be understood through a model wherein the $\mathrm{mPFC}$, and the dorsal anterior cingulate cortex (dACC) in particular, learns to predict and evaluate outcomes (Alexander and Brown, 2011; Silvetti et al., 2011, 2014). Within this framework, the role of the dACC is to compute prediction errors (PEs) that reflect the deviation between expected and received outcomes (Alexander and Brown, 2011).

Whereas our PE-related model implies a specifically cognitive role for the dACC, recent meta-analyses of fMRI studies suggest that the dACC is also engaged by negative affect and pain (Etkin et al., 2011; Shackman et al., 2011). These data have been interpreted as evidence that the AACC performs a common evaluative 


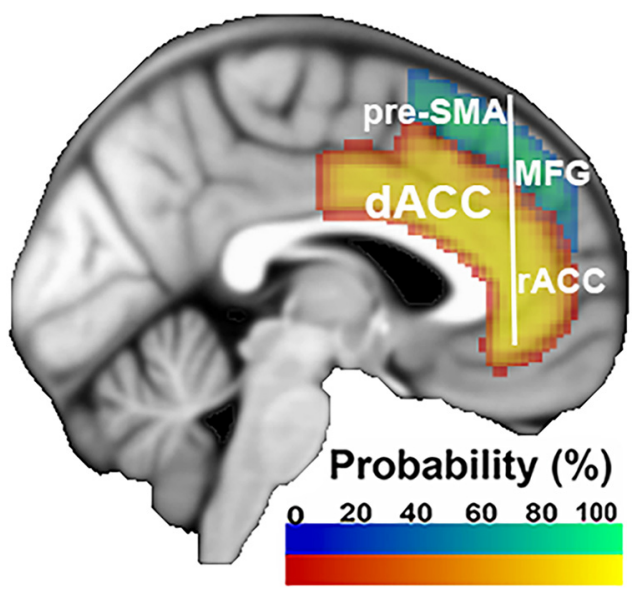

Figure 1. The pre-SMA, dACC, medial frontal gyrus (MFG), and rostral ACC ( $\mathrm{rACC}$ ) within the mPFC. Probabilistic maps of the cingulate gyrus (anterior division), paracingulate gyrus, and superior frontal gyrus were generated by the Harvard-0xford Atlas in FSL (Jenkinson et al., 2012), thresholded at $25 \%$ probability, and projected onto the ICBM152 template. For depiction purposes, the cingulate gyrus and paracingulate gyrus maps were added together to form the $\mathrm{AACC}$ map. The $\mathrm{dACC}$ is bounded dorsally by the cingulate sulcus in $\mathrm{pCgS}^{-}$subjects and the paracingulate sulcus in $\mathrm{pCgS}^{+}$subjects. Anteriorly, the $\mathrm{dACC}$ is divided from the $\mathrm{rACC}$ by the genu of the corpus callosum. Dorsal to the DACC is the pre-SMA, bounded posteriorly by a vertical line extending from the anterior commissure and bounded anteriorly by a vertical line extending from the genu of the corpus callosum (Picard and Strick, 2001; Kim et al., 2010).

or control role across different domains of input (cognition, emotion, pain). However, demonstrating common activation loci across multiple domains may mask the selectivity of a region to a given function. To investigate this possibility, Lieberman and Eisenberger (2015) performed reverse inference on the dACC with terms relating to pain and cognitive control (e.g., conflict). This approach allowed the authors to infer which of these phenomena is most associated with activation in the dACC. This analysis revealed that the AACC is preferentially associated with pain, whereas cognitive effects are better localized to more dorsal areas, such as the pre-SMA (Fig. 1). These data suggest that there is a dorsal-ventral distinction between cognitive processing and pain in the mPFC.

The claim that dACC is selective for pain has generated significant recent controversy with subsequent meta-analyses providing equivocal results (de la Vega et al., 2016; Lieberman et al., 2016; Wager et al., 2016). However, meta-analyses of these sorts have several limitations. First, direct comparison between phenomena can be biased if one phenomenon is over-represented (e.g., more studies of pain than conflict), or more consistently isolated due to superior or homogeneous experimental control. Second, meta-analyses can mask the effect of individual anatomical variability on the localization of specific effects. For example, subjects with a paracingulate sulcus ( $\mathrm{pCgS}$ ) show activation profiles that are more dorsal than subjects without a pCgS (Amiez et al., 2013). Furthermore, the ability to dissociate the roles of nearby regions through meta-analysis is undermined by using peak coordinates rather than the full extent of activations, which can mask areas of overlap. It would therefore be beneficial to directly compare cognitive and pain processing within a single well-matched procedure.

To that end, we created a procedure that orthogonally manipulated factors of pain, conflict, and their predictability. Based on our previous model (Alexander and Brown, 2011), we expected that conflict and PEs of conflict would activate the same area of the mPFC. Furthermore, we anticipated that PEs of pain would activate the same area of the MPFC as cognitive effects (Alexander and Brown, 2014). Whether pain itself would activate this same region or a distinct area was an exploratory matter aimed to resolve the recent debate.

\section{Materials and Methods}

Participants. Data from 29 right-handed participants (10 female) were collected (mean \pm SD age: $24.0 \pm 2.80$ years). Participants did not report a history of psychiatric or neurological disorder, and reported no current use of psychoactive medications. Participants were compensated $\$ 30 / \mathrm{h}$ for their time. Participants were trained on the task on a computer outside of the scanner for two practice blocks on a separate day from the scanning session. When they returned for the scanning session, participants performed one practice block of the task outside of the scanner before undergoing the scanning session. Data from two subjects were discarded due to insufficient accuracy ( $>3$ SDs below the mean accuracy of all subjects), and data from an additional subject were discarded due to a self-reported failure to follow the instructions, leaving a total of 26 useable participants $(n=26)$.

Electrical stimulation apparatus. To deliver electrical shock, a transcutaneous aversive finger stimulator was used (model E13-22, Coulbourn Instruments). The range of electrical shock delivered by the device ranged from 0.2 to $4.0 \mathrm{~mA}$, with nine discrete levels. MRI-compatible electrodes were placed on the fourth and fifth phalanges of the left hand. Before undergoing scanning, participants were administered the lowest possible level of electrical shock from the finger stimulator. The current was raised incrementally, and participants were instructed to tell the experimenter when the amount of current became aversive. This setting was used as their high level of electrical shock. Starting again from the lowest level of shock, participants were instructed to tell the experimenter when the level of shock was noticeable, but not aversive. This setting was used as their low level of electrical shock.

Because the sensitivity to shocks could increase or decrease over time, participants were asked whether either level of shock was too high or too low at the end of each experimental block. The levels of shock were then adjusted until the participant reported that both the high level and low level of electrical shock met the original criteria. Across all subjects, the lowest level chosen by participants was $0.5 \mathrm{~mA}$, whereas the highest level was $2.7 \mathrm{~mA}$. This is in line with previous studies using shock to elicit pain (e.g., Wager et al., 2004).

Galvanic skin response. Galvanic skin response (GSR) data were recorded to provide a physiological measurement of pain. These data were collected using the MP-150 system (BIOPAC Systems) at a sampling rate of $250 \mathrm{~Hz}$ by using MRI-compatible electrodes placed on the thenar and hypothenar of the left hand. GSR data were low pass-filtered, allowing frequencies $<15 \mathrm{~Hz}$ and detrended. The peak GSR value was then extracted within a window of $1-6 \mathrm{~s}$ after the administration of electrical stimulation or cognitive stimulus.

Procedure. The task (Fig. 2) was designed to compare PEs related to stimuli eliciting either cognitive control for a conflict task or processing an electrical shock (hereafter referred to as $\mathrm{PE}_{\mathrm{cog}}$ and $\mathrm{PE}_{\text {pain }}$, respectively), as well as to examine main effects of pain and conflict. We used predictive cues signaling both the level of aversion (high or low) and modality (cognitive or pain) of an upcoming stimulus. Cues were probabilistic such that some stimuli were more or less aversive than expected. Aversive pain was controlled by the level of electrical shock. For cognitive stimuli, on the other hand, there is evidence that more effortful processing is aversive in terms of cost of control (McGuire and Botvinick, 2010; Shenhav et al., 2013). Hence, aversion was controlled by the level of cognitive control required by varying response conflict. The design therefore allowed direct comparison of conflict, pain, and PEs for cognitive and pain stimuli, allowing us to test the relative sensitivity of mPFC subregions to these phenomena.

To achieve this, a 2 (modality) $\times 3$ (cue) factorial design was used. The factor of modality consisted of two levels: cognitive (i.e., the conflict trials) and pain (i.e., the electrical shocks). The factor of cue consisted of three levels: a cue signaling a $75 \%$ chance of obtaining a high-aversion outcome (incongruent spatial stimulus or high shock stimulus), a cue 


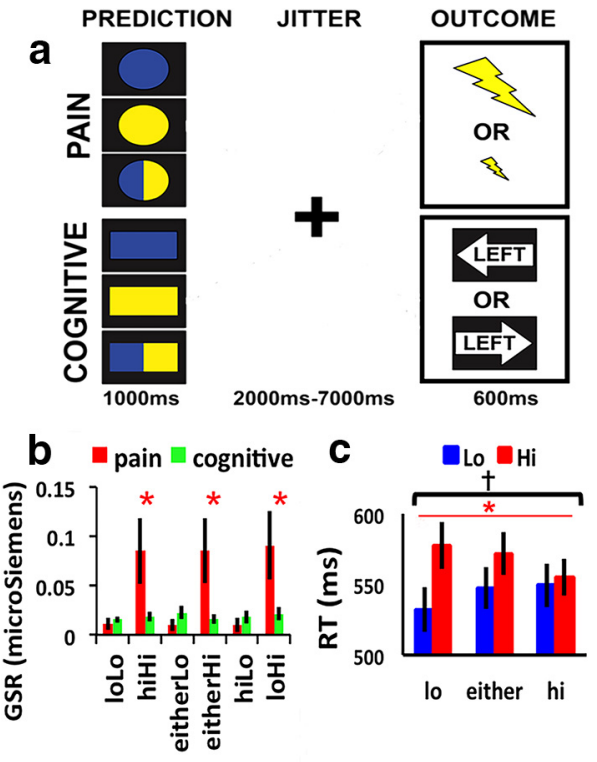

Figure 2. Procedure and behavioral results. $\boldsymbol{A}$, Task. During the prediction phase, subjects are presented with a cue signaling whether the outcome will be electrical shock or a spatial stimulus, as well as the probability of whether it will be a high aversion or low aversion outcome. Cues that are half blue and half yellow always signify that there is an equal chance of receiving either a more aversive or less aversive outcome; the probabilities signified by either blue or yellow cues were counterbalanced across subjects. Circles represent electrical shock condition. Rectangles represent spatial condition, The meaning of the shapes was also counterbalanced across subjects. The prediction phase is followed by a jittered interval, and then the outcome of either an electric shock or a spatial stimulus. $B$, GSR across pain and cognitive outcomes. C, RTs across conflict conditions. Error bars indicate SEM. * Main effect, $p<0.001$. tinteraction, $p<0.05$.

signaling a $75 \%$ chance of obtaining a low-aversion outcome (congruent spatial stimulus or low shock stimulus), and a cue signaling a $50 \%$ chance of obtaining either level of conflict or shock stimulus.

The task had two phases of interest: a prediction phase and an outcome phase. The prediction phase for each trial lasted for $1 \mathrm{~s}$, during which participants were presented with a cue to predict the outcome. This prediction phase was followed by a jittered interval of 2-7 s where the participants saw a fixation cross. This was then followed by an outcome phase lasting $600 \mathrm{~ms}$, during which either a spatial stimulus was presented or electrical shock was administered. The outcome phase for the cognitive condition consisted of either an incongruent spatial stimulus (e.g., the word "Left" printed inside of an arrow pointing to the right), or a congruent spatial stimulus (e.g., the word "Right" printed inside of an arrow pointing toward the right). The outcome phase for the pain condition consisted of either a high-aversion shock or a lowaversion shock. This was followed by another jittered interval of $4-8 \mathrm{~s}$ to allow for independent estimation of the BOLD signal of the outcome phase. This combination of cues and outcomes led to either expected outcomes or unexpected outcomes (i.e., PEs). For example, a PE for the pain condition would be receiving a cue predicting a low-level shock, and then during the outcome phase receiving a high-level shock (Fig. 2A).

In total, there were five runs of scanning per subject. Each run contained 36 cognitive trials and 36 pain trials, for a total of 180 trials for each modality per subject. There were an equal number of trials using highaversion predicting, low-aversion predicting, and uninformative cues. For the predictive cues, 25\% of the trial outcomes were PEs, whereas $75 \%$ of the outcomes were consistent with the predictive cue. There was an equal number of each type of outcome for uninformative cues. In total, for each modality, there were 15 of each type of PE.

Image acquisition and preprocessing. The experiment was conducted with a 3 tesla Siemens Trio scanner using a 32-channel head coil. Foam padding was inserted around the sides of the head to increase participant comfort and reduce head motion. Imaging data were acquired at a $30^{\circ}$ angle from the anterior commissure-posterior commissure line (Deich- mann et al., 2003). Functional T2* -weighted images were acquired using a gradient EPI sequence: $50 \times 2.7 \mathrm{~mm}$ interleaved slices; TE $=25 \mathrm{~ms}$; $\mathrm{TR}=3000 \mathrm{~ms} ; 96 \times 96$ voxel matrix; $220 \times 220 \mathrm{~mm}$ field of view; $2.29 \times$ 2.29 in-plane resolution. For the experimental condition, five runs of data were collected with 208 functional scans each. High-resolution T1-weighted images for anatomical data $(256 \times 256$ voxel matrix $)$ were collected at the end of each session.

Functional data were spike-corrected using AFNI's despiking algorithm (http://afni.nimh.nih.gov/afni; RRID:SCR_005927). SPM5 (Wellcome Department of Imaging Neuroscience, London; www.fil.ion.ucl. ac.uk/spm; RRID:SCR_007037) was used for subsequent preprocessing and data analysis. The functional data for each run for each participant were slice-time corrected and realigned to each run's mean functional image using a 6 degree-of-freedom rigid body spatial transformation. The resulting images were then coregistered to the participant's structural image. Structural data were coregistered to the functional data and segmented into gray and white-matter probability maps. These segmented images were used to calculate spatial normalization parameters to the ICBM152 template, which were subsequently applied to the functional data. As part of spatial normalization, the data were resampled to $2 \times 2 \times 2 \mathrm{~mm}$. The structural image was normalized to standard MNI space, and the warps were applied to the functional images. The functional images were then spatially smoothed using an $8 \mathrm{~mm}$ Gaussian kernel.

fMRI modeling and analysis. The combination of the cue, modality, and outcome factors outlined above led to 12 regressors coding for prediction-outcome pairs. To refer to these regressors, we begin with the predicted aversion level (lo/hi/either), followed by received aversion level (Lo/Hi), subscripted by the modality (pain/cog). This results in six outcome regressors in the pain condition $\left(\mathrm{loLo}_{\text {pain }}, \mathrm{loHi}_{\text {pain }}, \mathrm{hiLo}_{\text {pain }}\right.$, $\mathrm{hiHi}_{\text {pain }}$, eitherHi $\mathrm{i}_{\text {pain }}$, and eitherLo $\left.{ }_{\text {pain }}\right)$ and an additional six regressors for outcome in the conflict condition $\left(\mathrm{loLo}_{\mathrm{cog}}, \mathrm{loHi}_{\mathrm{cog}}, \mathrm{hiLo}_{\mathrm{cog}}, \mathrm{hiHi} \mathrm{i}_{\mathrm{cog}}\right.$, eitherHi $\mathrm{i}_{\mathrm{cog}}$, and eitherLo $\mathrm{o}_{\mathrm{cog}}$ ). We also included six prediction-related regressors (three related to pain trials and three related to cognitive trials) to capture prediction-related activity. Regressors were modeled with an impulse $\delta$ function at the time of onset convolved with the canonical hemodynamic response function implemented in SPM5. Both the onset of the prediction phase and the onset of the outcome phase were modeled separately. Motion regressors were included if the participant's motion exceeded $3 \mathrm{~mm} /$ degrees in any combination of translations or rotations of the head, or if there was any TR-to-TR motion $>0.5 \mathrm{~mm} /$ degrees. Separate regressors modeled the onset of the motor response for each trial. In addition, regressors modeling the reaction time (RT) for each trial were included to account for variability in the BOLD signal related to time on task (Grinband et al., 2008).

Contrasts were created to examine effects of pain, conflict, $\mathrm{PE}_{\mathrm{cog}}$, $\mathrm{PE}_{\text {pain }}$. Pain was assessed by contrasting high levels of shock with low levels of shock, collapsing across prediction conditions. Conflict, on the other hand, has been shown to be minimized by prediction and adaptation (Gratton et al., 1992; Aarts and Roelofs, 2011). We therefore tested for conflict by contrasting incongruent with congruent trials following congruent predicting cues (i.e., $\left.\left[\mathrm{loHi}_{\mathrm{cog}}-\mathrm{loLo}_{\mathrm{cog}}\right]\right)$. We note in passing that a main effect of conflict contrast that collapsed across levels of prediction did not reveal significant activations, which is consistent with previous work (Aarts and Roelofs, 2011). $\mathrm{PE}_{\mathrm{cog}}$ and $\mathrm{PE}_{\text {pain }}$ were assessed by contrasting conditions where predictions were violated against conditions where predictions were met: $[\mathrm{loHi}+$ hiLo $]-[\mathrm{loLo}+\mathrm{hiHi}]$. To maximize power for certain regions of interest (ROIs) and data-driven analyses, we created an additional modality-general PE contrast by collapsing PEs across pain and conflict conditions, referred to as PE. Significant overlap of activation between contrasts was tested using minimum conjunction analysis (Nichols et al., 2005), as well as within ROIs detailed below.

Unless otherwise stated, all voxelwise results were thresholded at an individual voxel level of $p<0.001$. Cluster extent thresholds corrected for multiple comparisons $(p<0.05)$ were calculated through AFNI's $3 \mathrm{dCl}$ ustSim. We used a version of AFNI compiled on June 30, 2016, which includes a spatial autocorrelation correction for cluster thresholds that were previously calculated to be too liberal (Eklund et al., 2016). 
Based on 3dClustSim, whole-brain analyses required a cluster size of at least 257 contiguous voxels (2-sided test, faces touching) to be significant. For more targeted analyses, we created an ROI of the mPFC based on the Harvard-Oxford Atlas distributed with FSL (http://fsl.fmrib.ox. ac.uk/fsl/), selecting the labels superior frontal gyrus, paracingulate gyrus, and cingulate gyrus (anterior division), thresholding each at 5\% probability and restricting the boundaries to MNI $-12<x<12,0<y<$ 60 , and $z>-15$. According to 3dClustSim, a cluster size of at least 29 voxels (2-sided test, faces touching) within the mask was required to be significant. At a more liberal primary threshold of $p<0.01$ uncorrected, the cluster size for significance within the mask $(p<0.05)$ was calculated to be 131 voxels. This more liberal threshold was used for a smallvolume-corrected analysis of conflict restricted to the levels of prediction described above wherein activations did not pass the more strict criteria. Although a primary threshold of $p<0.01$ has been shown to increase false positive rates (Eklund et al., 2016), false positives are somewhat less of a concern here since the contrast replicates a previously documented result with the same procedure (Aarts and Roelofs, 2011).

Leave-one-out analysis. To create data-driven, independent ROIs to examine each effect, we used a leave-one-out cross-validation method (Esterman et al., 2010). In this approach, second-level analyses are run for each contrast, consecutively leaving out each subject from the GLM and extracting that subject's contrast estimates from the resulting ROI. For this analysis, we used maps thresholded at the $p<0.01$ level and cluster-corrected at $p<0.05$.

Neurosynth ROI analysis. To complement our leave-one-out analysis, we created data-independent ROIs with Neurosynth (http://www. neurosynth.org). Meta-analysis maps were generated using forward inference for the terms "pain," "conflict," and "prediction error." The $5 \mathrm{~mm}$ spheres were created around the peak $z$-values of each of these maps within the $\mathrm{mPFC}$. Contrast estimates of PE, conflict, and pain were then extracted from these ROIs.

$\mathrm{k}$-means cluster analysis. To summarize sets of our univariate results, we conducted a $k$-means analysis, a data compression technique useful for summarizing several sets of contrasts simultaneously (Van Snellenberg et al., 2015). To restrict the focus of our analysis and reduce computational load, we used the mPFC mask described above and clustered over the contrast estimates from PE, conflict, and pain. Using the silhouette evaluation method in MATLAB (The MathWorks), the optimal number of clusters was determined to be 2 (Rousseeuw, 1987).

Morphological analysis. To better visualize our results with respect to individual anatomical variation, we averaged together the T1-weighted images of all individuals with a $\mathrm{pCgS}$ averaged over both hemispheres and projected the results of our morphological analysis onto this average anatomical image. To increase the power of this analysis, we used a mask of the mPFC (see above).

\section{Results}

\section{Behavioral results}

We began by examining whether GSR provided an overt signature of pain processing. As predicted, a paired $t$ test revealed a significantly higher GSR for high compared with low shocks (mean for $\mathrm{Hi}=0.87 \mathrm{mS}$; mean for Lo $=0.01 \mathrm{mS} ; t_{(22)}=2.79, p<$ $0.05)$. GSR did not differ between cognitive conditions (all paired $t<1.7 ; p>0.1$ ). Whereas high shocks led to greater GSR than cognitive stimuli $\left(t_{(22)}=4.97, p<0.001\right)$, low shocks elicited significantly lower GSR than cognitive stimuli $\left(t_{(22)}=-4.15, p<\right.$ $0.001)$. These data are consistent with the idea that high shocks elicit pain as opposed to low shocks. Finally, there was no interaction between predicted and received shocks $\left(F_{(2,44)}=0.93, p>\right.$ 0.1 ), indicating that prediction did not diminish the physiological effect of shocks (Fig. 2B).

Next, we examined cognitive effects on RT. Based on results from a previous study using a similar task (Aarts and Roelofs, 2011), we hypothesized that RTs would be slowed by outcomes inconsistent with cue predictions (i.e., PE) and would be faster when the cues were consistent with the outcome. To test this

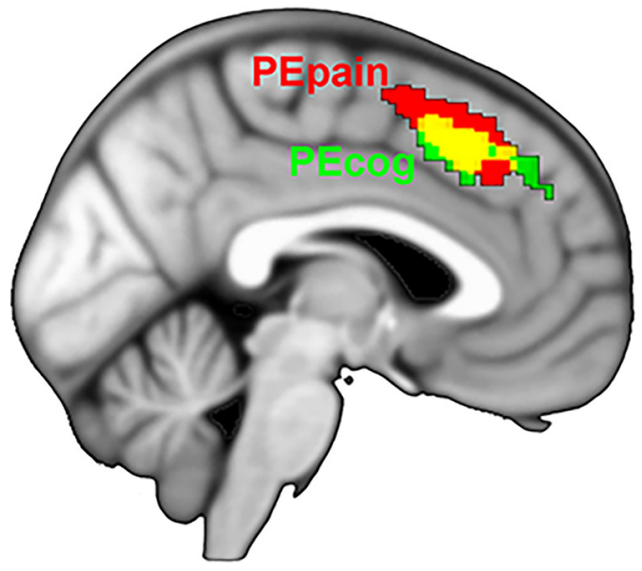

Figure 3. $P E_{\text {pain, }} \mathrm{PE}_{\text {cog, }}$ and their overlap (yellow). Results depicted at voxelwise threshold of $p<0.01$ uncorrected, $p<0.05$ cluster-corrected.

hypothesis, a 3 (predictive cue) $\times 2$ (outcome) ANOVA was performed. This analysis revealed a significant main effect of outcome driven by slowed responses to incongruent compared with congruent trials $\left(F_{(1,25)}=14.21, p<0.001\right)$. There was no main effect of prediction $\left(F_{(1,25)}=0.44, p=0.65\right)$. As predicted, there was a significant interaction between prediction and outcome $\left(F_{(2,50)}=5.25, p<0.01\right)$. The interaction was driven by faster RTs for outcomes that were consistent with predictions, and slower RTs for outcomes that were inconsistent with predictions (Fig. 2C).

\section{Imaging results}

Analysis of PEs

To examine the relative contribution of PEs within each modality and the location of their effects, we first looked at $\mathrm{PE}_{\mathrm{cog}}$ and $\mathrm{PE}_{\text {pain }}$ within a mask of the mPFC (see Materials and Methods). PE revealed significant clusters in the $\mathrm{mPFC}: \mathrm{PE}_{\text {pain }}, \mathrm{MNI}-4,20,48$; $k=60$ voxels; peak voxel $z$-value $=4.34 ; p<0.05$, clustercorrected; $\mathrm{PE}_{\mathrm{cog}}$, MNI 6, 18, 42; $k=243$ voxels; peak voxel $z$-value $=4.15, p<0.001$, cluster-corrected. These results show that $\mathrm{PE}$ in general is localized dorsally in the mPFC and that both $\mathrm{PE}_{\mathrm{cog}}$ and $\mathrm{PE}_{\text {pain }}$ contribute to this effect (Fig. 3).

Having shown a common neural substrate sensitive to both modalities of PE, we chose to focus subsequent analyses of PEs on an average of $\mathrm{PE}_{\mathrm{cog}}$ and $\mathrm{PE}_{\text {pain }}$, referred to hereafter as $\mathrm{PE}$ (see Materials and Methods). This fulfilled the double purpose of (1) maximizing power and (2) testing for a modalitygeneral effect of PE.

\section{Effects of pain and conflict}

We next tested for effects of pain and conflict. If a dorsal-ventral distinction exists within the MPFC, we would expect conflict to be represented more dorsally, similar to the location of our PE effects, and for pain to be localized more ventrally (Lieberman and Eisenberger, 2015). Consistent with this prediction, a significant main effect of pain was found within the cingulate gyrus of the dACC (MNI $-2,30,14 ; k=788$; peak $z$-value $=5.17 ; p<$ 0.001 , cluster-corrected; Fig. 4). In contrast, an analysis of conflict effects revealed a significant cluster more dorsally within the mPFC (MNI 0, 20, 44; $k=536$ voxels; peak $z$-value $=3.62 ; p<$ 0.05 , cluster-corrected). Together with our previous analysis of $\mathrm{PE}$, these results point toward a regional dissociation of pain and cognitive processing within the mPFC (Fig. 4). Other wholebrain effects are reported in Table 1. 

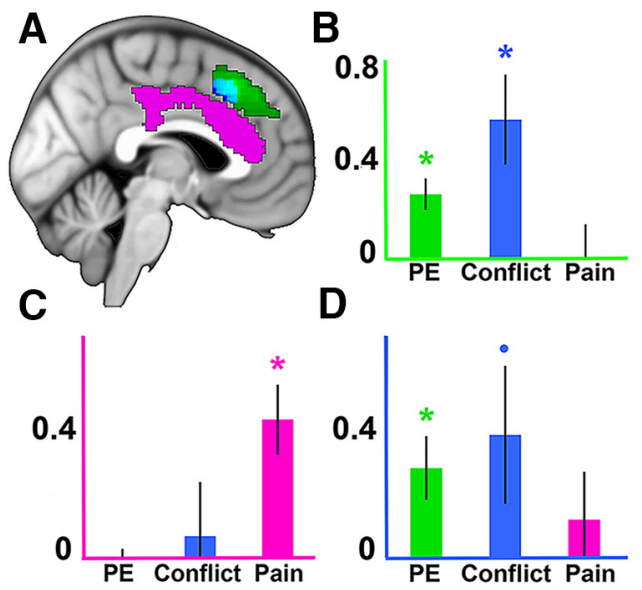

Figure 4. ROI results. $\boldsymbol{A}$, All whole-brain effects depicted at a voxelwise threshold of $p<$ 0.01 , cluster-corrected at $p<0.05$. Green represents PE. Blue represents conflict. Magenta represents pain. Cyan represents overlap of $P E$ and conflict. $\boldsymbol{B}-\boldsymbol{D}$, Results from $\mathrm{ROIs}$ from the leave-one-out procedure (Esterman et al., 2010) defined by PE (B), pain (C), and conflict $(\boldsymbol{D})$. Axes are color-coded to reflect their associated ROI. Error bars indicate SEM. ${ }^{*} p<0.01 .{ }^{\bullet} p<0.1$.

Table 1. Whole-brain activations at $p<0.001$ uncorrected, $p<0.05$ clustercorrected

\begin{tabular}{lrrrrrr}
\hline Brain region & $x$ & $y$ & $z$ & Zscore & $\begin{array}{l}\text { Cluster-corrected } \\
p \text { value }\end{array}$ & $\begin{array}{l}\text { Cluster size } \\
\text { (voxels) }\end{array}$ \\
\hline $\begin{array}{l}\text { Pain } \\
\text { Right somatosensory }\end{array}$ & 38 & -24 & 54 & 5.91 & $<0.001$ & 762 \\
$\quad \begin{array}{l}\quad \text { cortex } \\
\text { Right insula }\end{array}$ & 40 & -18 & 12 & 5.76 & $<0.001$ & 902 \\
$\begin{array}{l}\text { Left cerebellum } \\
\text { Left cingulate gyrus }\end{array}$ & -28 & -56 & -24 & 5.43 & $<0.001$ & 832 \\
$\begin{array}{l}\text { Right cerebellum } \\
\text { Right pariet0-0ccipital }\end{array}$ & 2 & -70 & 14 & 5.17 & $<0.001$ & 788 \\
$\quad$ sulcus & 8 & -84 & 40 & 3.85 & $<0.001$ & 335 \\
\hline
\end{tabular}

\section{Separate regions of $\mathrm{mPFC}$ process pain and cognitive effects}

Thus far, we have identified different regions of mPFC sensitive to cognitive and pain effects. To directly compare these effects, we used ROIs created with a leave-one-out procedure (see Materials and Methods) to test for regional dissociations. This was accomplished via a 3 (contrast) $\times 3$ (ROI) ANOVA. Consistent with the idea of heterogeneity within the mPFC, we found a significant contrast $\times$ ROI interaction $\left(F_{(4,100)}=11.33, p<0.001\right)$ and significant main effects of ROI for $\mathrm{PE}\left(F_{(2,75)}=4.26, p<0.05\right)$ and pain $\left(F_{(2,75)}=4.10, p<0.05\right)$. These results were driven by stronger conflict and PE effects and weaker pain effects in the dorsal ROIs, whereas this pattern was reversed in the ventral ROI. Within the PE ROI, both PE and conflict were significant (PE: $t_{(25)}=4.10, p<0.001$; conflict: $t_{(25)}=3.11, p<0.01$ ), whereas pain was not significant $\left(t_{(25)}=0.04, p>0.1\right)$. Within the conflict ROI, PE was significant $\left(t_{(25)}=2.85, p<0.01\right)$ and conflict trended toward significance $\left(t_{(25)}=1.79, p=0.09\right)$, whereas pain was not significant $\left(t_{(25)}=0.81, p>0.1\right)$. Last, within the pain ROI, pain was significant $\left(t_{(25)}=3.98, p<0.001\right)$, whereas neither PE nor conflict was significant $\left(\mathrm{PE}: t_{(25)}=-0.61, p>0.1\right.$; conflict: $t_{(25)}=0.41, p>0.1$ ). These results suggest that a more dorsal region of the $\mathrm{mPFC}$ represents cognitive processing, whereas a ventral region of the dACC processes pain (Fig. 4).

To examine the consistency of our results with other studies, we used data-independent ROIs created with Neurosynth (see Materials and Methods). This analysis replicated the results of
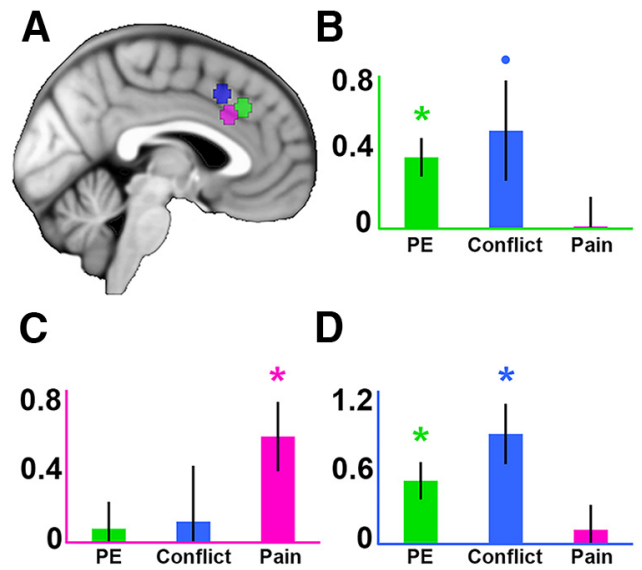

Figure 5. ROI results using Neurosynth. $\boldsymbol{A}$, Depiction of $5 \mathrm{~mm}$ ROls placed around peak coordinates of forward-inference meta-analysis maps of "prediction error" (green), "conflict" (blue), and "pain" (magenta). B-D, Extracted contrast estimates for PE, conflict, and pain; axes are color-coded to reflect their associated ROI. Error bars indicate SEM. ${ }^{*} p<0.01 .{ }^{\bullet} p<0.1$.

our leave-one-out analysis by revealing a significant contrast $X$ ROI interaction $\left(F_{(4,100)}=6.36, p<0.001\right)$. Similar to our previous analysis, this interaction was driven by larger cognitive effects and weaker pain effects in the conflict and PE ROIs, whereas this pattern reversed in the pain ROI. The PE ROI showed a significant effect of $\mathrm{PE}\left(t_{(25)}=3.69, p<0.01\right)$ and a trend toward significance for conflict $\left(t_{(25)}=1.95, p=0.06\right)$, whereas the conflict ROI showed significant effects for both $\mathrm{PE}\left(t_{(25)}=3.36\right.$, $p<0.01)$ and conflict $\left(t_{(25)}=3.65, p<0.01\right)$. Pain effects were not significant in the PE ROI $\left(t_{(25)}=0.07, p>0.1\right)$ or the conflict ROI $\left(t_{(25)}=0.57, p>0.1\right)$. The pain ROI showed a significant effect of pain $\left(t_{(25)}=3.06, p<0.01\right)$ but not PE $\left(t_{(25)}=0.51, p>\right.$ $0.1)$ or conflict $\left(t_{(25)}=0.38, p>0.1\right)$. This suggests that our results are in line with other studies and that there is a significant regional dissociation between pain and cognitive effects (Fig. 5).

\section{Data-driven dissociation of pain and cognitive effects}

To provide further evidence of a regional dissociation of pain and cognition, we turned to data-driven methods. Specifically, we conducted a $k$-means analysis to parcel the mPFC into subregions as a function of sensitivities to pain, conflict, and PE (see Materials and Methods). This analysis revealed 2 clusters split primarily into distinct dorsal-ventral regions within the mPFC.

To test whether these clusters represented the same dorsalventral distinction reported in the univariate results, contrast estimates from each cluster were extracted for PE, conflict, and pain. Within the dorsal cluster, there were significant effects for $\mathrm{PE}\left(t_{(25)}=3.05, p<0.01\right)$ and conflict $\left(t_{(25)}=3.13, p<0.01\right)$, whereas in the ventral cluster, there was a significant effect of pain $\left(t_{(25)}=2.33, p<0.05\right)$. To formally test for a dissociation, a 2 (cluster) $\times 3$ (condition) ANOVA was performed, which revealed a significant interaction $\left(F_{(2,50)}=11.08, p<0.001\right)$. These results support the claim that cognitive and pain processes show a significant dorsal-ventral dissociation within the mPFC (Fig. 6).

\section{Morphological analysis}

Recent studies have shown that anatomical differences within the mPFC can affect the location of activation profiles for certain cognitive phenomena, such as error feedback (Amiez et al., 2013). In particular, the location of these activations has been shown to depend on whether the subject has a $\mathrm{pCgS}$, an additional sulcus running dorsal to and approximately parallel with 


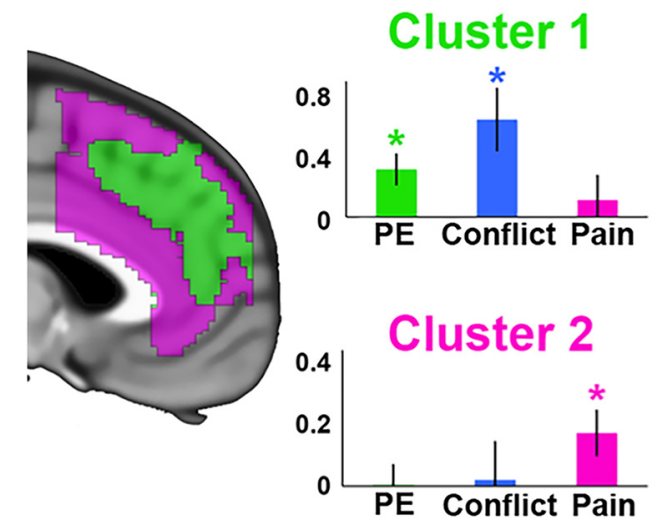

Figure 6. $k$-means cluster analysis performed with 2 clusters. Green represents Cluster 1. Magenta represents Cluster 2. Contrast estimates are plotted within each cluster for PE, conflict, and pain. Error bars indicate SEM. ${ }^{*} p<0.01$.

the cingulate sulcus. In subjects with a pCgS, dACC activations may appear to be within the pre-SMA in a canonical template of a single subject, such as the Colin N27 brain (Holmes et al., 1998). Hence, although our group-level analyses suggested that PE is localized to the same region across subjects, it is important to determine whether such localization is dependent upon individual differences in morphology.

To test this possibility, we categorized participants according to whether they had a $\mathrm{pCgS}\left(\mathrm{pCgS}^{+}\right)$or not $\left(\mathrm{pCgS}^{-}\right)$in either the left or right hemisphere. A subject was determined to have a $\mathrm{pCgS}$ if they showed a sulcus running dorsal to the cingulate sulcus for at least $25 \mathrm{~mm}$ and for at least 3 contiguous sagittal slices (Fornito et al., 2008). Eighteen subjects (69\% of our sample) had at least one $\mathrm{pCgS}$, a percentage that is consistent with prior studies (Paus et al., 1996). Ten of these subjects had a pCgS only in the right hemisphere, 4 subjects had one only in the left hemisphere, and 6 subjects had a $\mathrm{pCgS}$ in both the left and right hemispheres. $t$ tests of $\mathrm{PE}$ for each group within each hemisphere revealed no significant effects (all $p$ values $>0.1$ ), even after restricting our analysis to an mPFC mask (see Materials and Methods). To increase the power of our analysis, we restricted our analysis to the mPFC mask and flipped the orientation of each subject's right hemisphere to map onto each subject's left hemisphere (Amiez et al., 2013). This mapped an equal number of $\mathrm{pCgS}^{+}$and $\mathrm{pCgS}^{-}$contrast images (26 in each group) onto a single hemisphere.

After this averaging, we then tested for effects of PE within each group. This revealed activation in the pre-SMA in both groups $\left(\mathrm{pCgS}^{+}\right.$subjects: $\mathrm{MNI}-2,24,44 ; k=137$ voxels; $p<$ 0.05 , cluster-corrected; $\mathrm{pCgS}^{-}$subjects: $\mathrm{MNI}-2,22,46 ; k=182$ voxels; $p<0.05$, cluster-corrected), but dACC only in $\mathrm{pCgS}^{+}$ participants. However, at a reduced threshold of a voxelwise $p=$ 0.05 , activation extended from the pre-SMA into the cingulate sulcus in the $\mathrm{pCgS}^{-}$group, suggesting that any group discrepancies are most likely a result of statistical power and thresholding (Fig. 7A); conjunction analysis revealed significant overlap between the two clusters (MNI $-6,20,42 ; k=47$ voxels; $p<0.05$, cluster-corrected; Fig. 7). Previous work has reported that PErelated activations are observed in the cingulate sulcus in individuals without a pCgS (Amiez et al., 2012, 2013). In those studies, $\mathrm{pCgS}^{-}$individuals also demonstrated robust activations in the pre-SMA. When adopting a more liberal $p<0.05$ primary threshold consistent with Amiez et al. (2012), we found that our cluster extended across the pre-SMA and into the cingulate sulcus, demonstrating consistency between these studies. These re- sults suggest that PEs show similar patterns of activity across the pre-SMA and dorsal boundary of the dACC across subjects, regardless of the presence or absence of a pCgS.

\section{Discussion}

In this study, we examined whether pain and cognitive processing are represented in distinct or overlapping regions of the $\mathrm{mPFC}$. We found a double dissociation within the mPFC between the cognitive processes of PE and conflict located more dorsally and pain represented more ventrally, consistent with the metaanalysis results reported by Lieberman and Eisenberger (2015). These results are in contrast to meta-analyses suggesting that these processes overlap (Shackman et al., 2011; de la Vega et al., 2016) or are dissociable between the dACC and the rostral ACC (Bush et al., 2000; Etkin et al., 2011). Analyses of subjects classified by the presence or absence of a $\mathrm{pCgS}$ showed that both groups had PE activation in both the dACC and pre-SMA.

\section{Contributions of individual anatomical variability}

One major limitation of meta-analyses is the averaging together of individuals with significant variations in cortical anatomy (Lieberman and Eisenberger, 2015; Wager et al., 2016; de la Vega et al., 2016). In the mPFC in particular, a major anatomical landmark, the pCgS, can change the localization of activity in response to feedback (Amiez et al., 2013; Amiez and Petrides, 2014). It is important to note that, within $\mathrm{pCgS}^{+}$subjects, the cingulate gyrus, paracingulate gyrus, and their corresponding sulci make up the dACC (Palomero-Gallagher et al., 2009).

The presence or absence of a pCgS defines the boundary between the dACC and the pre-SMA. In $\mathrm{pCgS}^{+}$subjects, activity dorsal to the $\mathrm{pCgS}$ is considered to fall within the pre-SMA region, whereas within $\mathrm{pCgS}^{-}$subjects, activity dorsal to the cingulate sulcus is considered pre-SMA (Kim et al., 2010). Furthermore, the presence of a $\mathrm{pCgS}$ increases the amount of cortical volume of the dACC relative to that of the pre-SMA (Fig. 7). Activity from a group average may consequently fall within the dACC for $\mathrm{pCgS}^{+}$subjects while being within the preSMA for $\mathrm{pCgS}^{-}$subjects. Therefore, to make strong claims about the location of effects, anatomical variability must be taken into account.

We addressed this in the current study by grouping subjects based on the presence or absence of a $\mathrm{pCgS}$. This revealed that both $\mathrm{pCgS}^{+}$and $\mathrm{pCgS}^{-}$subjects had overlapping activity within the mPFC. However, viewing each group's results on their corresponding average anatomical image showed $\mathrm{PE}$ effects within the pCgS and pre-SMA for $\mathrm{pCgS}^{+}$subjects, whereas PE was found within the pre-SMA (and at a lowered threshold, the cingulate sulcus) for the $\mathrm{pCgS}^{-}$group. Therefore, meta-analyses are correct in saying that cognitive effects are, on average, within the dACC. However, accounting for individual anatomical variability provides more sensitivity in determining where these effects are relative to pain processing. For example, a recent metaanalysis using $k$-means clustering found a cluster encompassing the dACC and pre-SMA that was associated with both cognitive and pain processing (de la Vega et al., 2016). However, post hoc tests revealed that the dorsal subregion was more strongly associated with the cognitive process of working memory, whereas the ventral subregion was associated with pain and affect. These findings are consistent with our results, which suggest that the distinction in this meta-analysis would become more pronounced by including anatomical variability. In previous studies of anatomical variability, feedback-related activity was found in the dACC: within the $\mathrm{pCgS}$ for $\mathrm{pCgS}^{+}$subjects and within the 

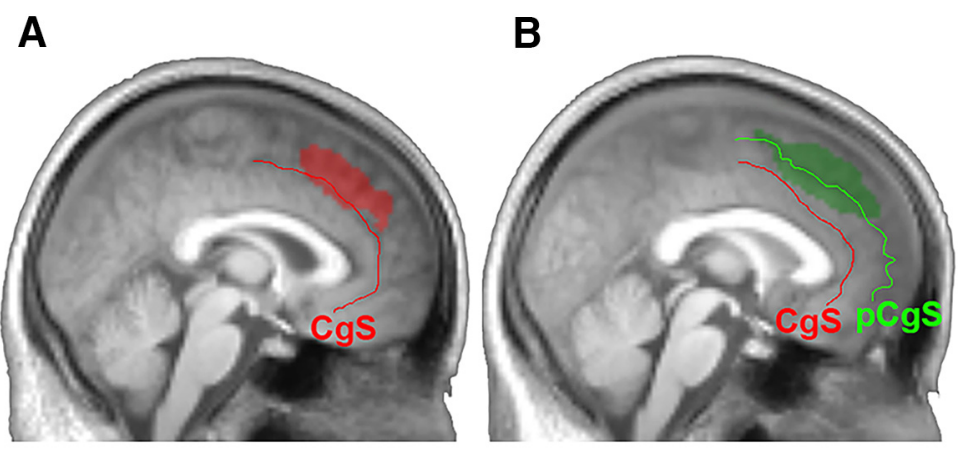

C

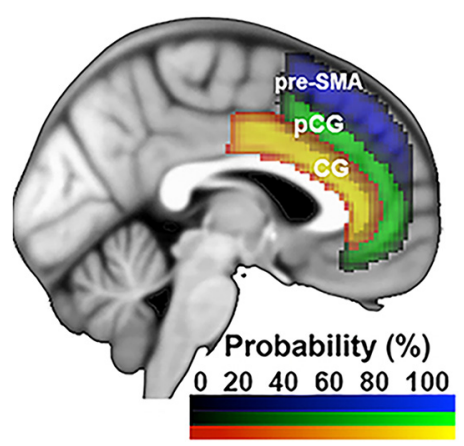

D

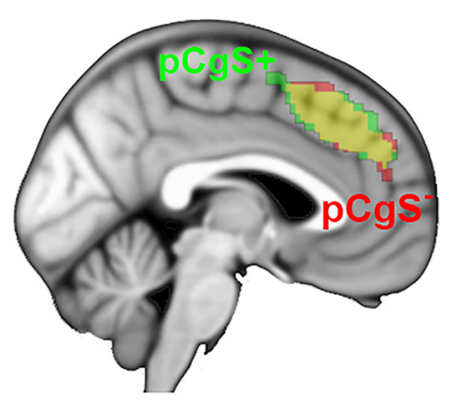

Figure 7. Effects of $\mathrm{PE}$ for $\mathrm{pCgS}{ }^{-}$and $\mathrm{pCgS}{ }^{+}$groups. Contrast maps were projected onto a pooled average of anatomical scans across hemispheres for each group. $A$, Location of PE effects for the $p \mathrm{CgS}^{-}$group at sagittal slice $x=0 . \boldsymbol{B}$, Location of PE effects for the $\mathrm{PCSS}^{+}$group at sagittal slice $x=-2 . \boldsymbol{A}, \boldsymbol{B}, p=0.05$ (uncorrected), showing spread of activation. $\boldsymbol{C}$, Depiction of subregions of the $\mathrm{mPFC}$ projected onto the ICBM152 thresholded at $25 \%$ probability. CG, Cingulate gyrus; $\mathrm{pCG}$, paracingulate gyrus. D, 0verlap of PE effects for $\mathrm{pCgS}^{-}$and $\mathrm{pCgS}^{+}$groups depicted at $p<0.05$ uncorrected, $p<0.05$ cluster-corrected.

region of the dACC appears to be more responsive to pain instead of cognitive processing, cognitive processes recruit both the pre-SMA and the dorsal area of the dACC.

In addition to addressing theories of $\mathrm{mPFC}$ heterogeneity, our results are also relevant to computational theories of the mPFC, such as the PRO model (Alexander and Brown, 2011). According to this model, the mPFC computes PEs regardless of valence. In the current study, PEs were generated across two different modalities (pain and conflict) and for both more and less aversive outcomes, suggesting that the mPFC computes a valencefree PE signal across different modalities (Jessup et al., 2010; Hayden et al., 2011).

\section{Potential Limitations}

Although GSR responses suggest that participants were feeling pain, no subjective ratings of pain were collected about whether they were actually experiencing pain. Our results should be read with this caveat in mind. In addition, as our study applied pain only to the hand, we cannot say how our results of pain and its PE would change if pain were applied elsewhere. For example, both motor activity and pain processing of the hand have been localized to the same region (Misra and

cingulate sulcus for $\mathrm{pCgS}^{-}$subjects (Amiez et al., 2013). Our results support this claim but also show that $\mathrm{PE}$ extends to the pre-SMA in $\mathrm{pCgS}^{-}$and $\mathrm{pCgS}^{+}$subjects (Amiez et al., 2012), whereas pain in both groups is localized within the cingulate gyrus (Fig. 7).

\section{Distinguishing between theories of mPFC function}

Our results address two theories of whether cognitive and pain processing occurs in distinct or similar regions of the mPFC: the neural alarm hypothesis (Lieberman and Eisenberger, 2015) and the adaptive control hypothesis (Shackman et al., 2011). According to the neural alarm hypothesis, the phylogenetically older dACC is responsive to events that concern the organism's survival. The pre-SMA, in contrast, is a relatively newer structure that has been implicated in more complex, arbitrary tasks, and is more responsive to cognitive processes as opposed to pain processing. Within this framework, the dACC is associated with stimuli related to immediate survival goals, whereas the pre-SMA is associated with cognitive processing.

According to the adaptive control hypothesis, on the other hand, the dACC processes a variety of aversive stimuli, including pain, negative affect, and stimuli, signaling the need for cognitive control. The dACC then sends signals to appropriate motor centers for executing goal-directed behavior, or to the frontoparietal cortex for adjudicating conflict (Shackman et al., 2011; Cavanagh and Shackman, 2015). To the extent that there is uncertainty about actions or outcomes, this theory posits that the dACC acts as a hub that responds to aversive stimuli regardless of modality. Our finding of a dorsal-ventral dissociation between cognitive and pain processing is in line with the neural alarm hypothesis. However, this comes with the caveat that, although the ventral
Coombes, 2015), and a meta-analysis of human and primate studies has shown that motor and feedback processing for different effectors is colocalized within different areas of the mPFC (Procyk et al., 2016). These results suggest that pain, motor, and feedback (e.g., PE) processing for different effectors would recruit different areas of the mPFC. Future work is needed to test whether the same regional dissociation reported in this study between pain and PE would be observed for different effectors. Furthermore, linking PE to an overt motor response within the same effector, a link that was not present in the current study, may change the presence of such a dissociation.

We also note that, although our experiment was designed to match the different conditions on probability of predicted outcomes, the cognitive modality included motor responses whereas the pain condition did not. However, because motor responses were modeled, any activity seen for PEs in the cognitive condition was likely not due to motor responses. Furthermore, the overlap between PEs of the different modalities suggests that PE processing is not significantly affected by motor activity.

In conclusion, by combining cognitive and pain processing in a single within-subjects experiment, this study provides empirical evidence directly related to the current debate about the organization of the mPFC. Overall, our findings show a dorsal-ventral division of processing within the $\mathrm{MPFC}$, with cognitive processing associated with the more dorsal aspect of the MPFC and pain processing localized more ventrally. Our separation of subjects according to the presence or absence of a pCgS showed similar functional profiles for $\mathrm{PE}$, but with some variability: $\mathrm{pCgS}^{+}$subjects showed PE-related activity within the paracingulate gyrus and pre-SMA, whereas $\mathrm{pCgS}^{-}$subjects showed PE effects mostly within the pre-SMA, but spreading into the cingulate sulcus at a 
reduced threshold. These results suggest that future studies should take individual anatomical variability into account, especially when examining cognitive effects within the mPFC.

\section{References}

Aarts E, Roelofs A (2011) Attentional control in anterior cingulate cortex based on probabilistic cueing. J Cogn Neurosci 23:716-727. CrossRef Medline

Alexander WH, Brown JW (2011) Medial prefrontal cortex as an actionoutcome predictor. Nat Neurosci 14:1338-1344. CrossRef Medline

Alexander WH, Brown JW (2014) A general role for medial prefrontal cortex in event prediction. Front Comp Neurosci 8:69. CrossRef Medline

Amiez C, Petrides M (2014) Neuroimaging evidence of the anatomofunctional organization of the human cingulate motor areas. Cereb Cortex 24:563-578. CrossRef Medline

Amiez C, Sallet J, Procyk E, Petrides M (2012) Modulation of feedback related activity in the rostral anterior cingulate cortex during trial and error exploration. Neuroimage 63:1078-1090. CrossRef Medline

Amiez C, Neveu R, Warrot D, Petrides M, Knoblauch K, Procyk E (2013) The location of feedback-related activity in the midcingulate cortex is predicted by local morphology. J Neurosci 33:2217-2228. CrossRef Medline

Behrens TE, Woolrich MW, Walton ME, Rushworth MF (2007) Learning the value of information in an uncertain world. Nat Neurosci 10:12141221. CrossRef Medline

Botvinick MM, Braver TS, Barch DM, Carter CS, Cohen JD (2001) Conflict monitoring and cognitive control. Psychol Rev 108:624-652. CrossRef Medline

Brown JW, Braver TS (2005) Learned predictions of error likelihood in the anterior cingulate cortex. Science 307:1118-1121. CrossRef Medline

Bush G, Luu P, Posner MI (2000) Cognitive and emotional influences in anterior cingulate cortex. Trends Cogn Sci 4:215-222. CrossRef Medline

Cavanagh JF, Shackman AJ (2015) Frontal midline theta reflects anxiety and cognitive control: meta-analytic evidence. J Physiol Paris 109:3-15. CrossRef Medline

de la Vega A, Chang JL, Banich MT, Wager TD, Yarkoni T (2016) Largescale meta-analysis of human medial frontal cortex reveals tripartite functional organization. J Neurosci 36:6553-6562. CrossRef Medline

Deichmann R, Gottfried JA, Hutton C, Turner R (2003) Optimized EPI for fMRI studies of the orbitofrontal cortex. Neuroimage 19:430-441. CrossRef Medline

Eklund A, Nichols TE, Knutsson H (2016) Cluster failure: why fMRI inferences for spatial extent have inflated false-positive rates. Proc Natl Acad Sci U S A 113:7900-7905. CrossRef Medline

Esterman M, Tamber-Rosenau BJ, Chiu YC, Yantis S (2010) Avoiding nonindependence in fMRI data analysis: leave one subject out. Neuroimage 50:572-576. CrossRef Medline

Etkin A, Egner T, Kalisch R (2011) Emotional processing in anterior cingulate and medial prefrontal cortex. Trends Cogn Sci 15:85-93. CrossRef Medline

Fornito A, Wood SJ, Whittle S, Fuller J, Adamson C, Saling MM, Velakoulis D, Pantelis C, Yücel M (2008) Variability of the paracingulate sulcus and morphometry of the medial frontal cortex: associations with cortical thickness, surface area, volume, and sulcal depth. Hum Brain Mapp 29: 222-236. CrossRef Medline

Gehring WJ, Goss B, Coles MG, Meyer DE, Donchin E (1993) A neural system for error-detection and compensation. Psychol Sci 4:385-390. CrossRef

Gratton G, Coles MG, Donchin E (1992) Optimizing the use of information: strategic control of activation of responses. J Exp Psychol Gen 121: 480-506. CrossRef Medline

Grinband J, Wager TD, Lindquist M, Ferrera VP, Hirsch J (2008) Detection of time-varying signals in event-related fMRI designs. Neuroimage 43: 509-520. CrossRef Medline

Grinband J, Savitskaya J, Wager TD, Teichert T, Ferrera VP, Hirsch J (2011) The dorsal medial frontal cortex is sensitive to time on task, not response conflict or error likelihood. Neuroimage 57:303-311. CrossRef Medline

Hayden BY, Pearson JM, Platt ML (2011) Neuronal basis of sequential foraging decisions in a patchy environment. Nat Neurosci 14:933-939. CrossRef Medline
Holmes CJ, Hoge R, Collins L, Woods R, Toga AW, Evans AC (1998) Enhancement of MR images using registration for signal averaging. J Comput Assist Tomogr 22:324-333. CrossRef Medline

Jenkinson M, Beckmann CF, Behrens TE, Woolrich MW, Smith SM (2012) Fsl. Neuroimage 62:782-790. CrossRef Medline

Jessup RK, Busemeyer JR, Brown JW (2010) Error effects in anterior cingulate cortex reverse when error likelihood is high. J Neurosci 30:3467-3472. CrossRef Medline

Kim JH, Lee JM, Jo HJ, Kim SH, Lee JH, Kim ST, Seo SW, Cox RW, Na DL, Kim SI, Saad ZS (2010) Defining functional SMA and pre-SMA subregions in human MFC using resting state fMRI: Functional connectivity-based parcellation method. Neuroimage 49:2375-2386. CrossRef Medline

Lieberman MD, Eisenberger NI (2015) The dorsal anterior cingulate cortex is selective for pain: results from large-scale reverse inference submission. Proc Natl Acad Sci U S A 112:15250-15255. CrossRef Medline

Lieberman MD, Burns SM, Torre JB, Eisenberger NI (2016) Reply to Wager et al.: Pain and the dACC: the importance of hit rate-adjusted effects and posterior probabilities with fair priors. Proc Natl Acad Sci U S A 113:1-4. CrossRef Medline

McGuire JT, Botvinick MM (2010) Prefrontal cortex, cognitive control, and the registration of decision costs. Proc Natl Acad Sci U S A 107:79227926. CrossRef Medline

Misra G, Coombes SA (2015) Neuroimaging evidence of motor control and pain processing in the human midcingulate cortex. Cereb Cortex 25: 1906-1919. CrossRef Medline

Nichols T, Brett M, Andersson J, Wager T, Poline JB (2005) Valid conjunction inference with the minimum statistic. Neuroimage 25:653660. CrossRef Medline

Palomero-Gallagher N, Vogt BA, Schleicher A, Mayberg HS, Zilles K (2009) Receptor architecture of human cingulate cortex: evaluation of the four-region neurobiological model. Hum Brain Mapp 30:2336-2355. CrossRef Medline

Paus T, Tomaiuolo F, Otaky N, MacDonald D, Petrides M, Atlas J, Morris R, Evans AC (1996) Human cingulate and paracingulate sulci: pattern, variability, asymmetry, and probabilistic map. Cereb Cortex 6:207-214. CrossRef Medline

Picard N, Strick PL (2001) Imaging the premotor areas. Curr Opin Neurobiol 11:663-672. CrossRef Medline

Procyk E, Wilson CR, Stoll FM, Faraut MC, Petrides M, Amiez C (2016) Midcingulate motor map and feedback detection: converging data from humans and monkeys. Cereb Cortex 26:467-476. CrossRef Medline

Rainville P, Duncan GH, Price DD, Carrier B, Bushnell MC (1997) Pain affect encoded in human anterior cingulate but not somatosensory cortex. Science 277:968-971. CrossRef Medline

Rousseeuw PJ (1987) Silhouettes: a graphical aid to the interpretation and validation of cluster analysis. J Comput Appl Math 20:53-65. CrossRef

Shackman AJ, Salomons TV, Slagter HA, Fox AS, Winter JJ, Davidson RJ (2011) The integration of negative affect, pain and cognitive control in the cingulate cortex. Nat Rev Neurosci 12:154-167. CrossRef Medline

Shenhav A, Botvinick MM, Cohen JD (2013) The expected value of control: an integrative theory of anterior cingulate cortex function. Neuron 79: 217-240. CrossRef Medline

Silvetti M, Seurinck R, Verguts T (2011) Value and prediction error in medial frontal cortex: integrating the single-unit and systems levels of analysis. Front Hum Neurosci 5:75. CrossRef Medline

Silvetti M, Nuñez Castellar E, Roger C, Verguts T (2014) Reward expectation and prediction error in human medial frontal cortex: an EEG study. Neuroimage 84:376-382. CrossRef Medline

Van Snellenberg JX, Slifstein M, Read C, Weber J, Thompson JL, Wager TD, Shohamy D, Abi-Dargham A, Smith EE (2015) Dynamic shifts in brain network activation during supracapacity working memory task performance. Hum Brain Mapp 36:1245-1264. CrossRef Medline

Wager TD, Rilling JK, Smith EE, Sokolik A, Casey KL, Davidson RJ, Kosslyn SM, Rose RM, Cohen JD (2004) Placebo-induced changes in fMRI in the anticipation and experience of pain. Science 303:1162-1167. CrossRef Medline

Wager TD, Atlas LY, Botvinick MM, Chang LJ, Coghill RC, Davis KD, Iannetti GD, Poldrack RA, Shackman AJ, Yarkoni T (2016) Pain in the ACC? Proc Natl Acad Sci U S A 113:E2474-E2475. CrossRef Medline 